\title{
ON A CLASS OF UNIVALENT FUNCTIONS DEFINED BY SǍLǍGEAN INTEGRO-DIFFERENTIAL OPERATOR
}

\author{
Á. O. PÁLL-SZABÓ
}

Received 22 November, 2017

\begin{abstract}
In this paper we consider the $\mathscr{L}^{n}: \mathcal{A}_{\rightarrow} \rightarrow \mathcal{A}$, $\mathscr{L}^{n} f(z)=(1-\lambda) \mathscr{D}^{n} f(z)+\lambda I^{n} f(z)$ linear operator, where $\mathscr{D}^{n}$ is the Sălăgean differential operator and $I^{n}$ is the Sălăgean integral operator. We study several differential subordinations generated by $\mathscr{L}^{n}$. We introduce a class of holomorphic functions $L_{n}^{m}(\beta)$, and obtain some subordination results.
\end{abstract}

2010 Mathematics Subject Classification: 30C45; 30A20; 34A40

Keywords: analytic functions, convex function, Sălăgean integro-differential operator, differential operator, differential subordination, dominant, best dominant

\section{Preliminaries}

Let $U$ be the unit disk in the complex plane:

$$
U=\{z \in \mathbb{C}:|z|<1\} .
$$

Let $\mathscr{H}(U)$ be the space of holomorphic functions in $U$ and let

$$
\mathcal{A}_{m}=\left\{f \in \mathscr{H}(U): f(z)=z+a_{m+1} z^{m+1}+\cdots, z \in U\right\}
$$

with $\mathcal{A}_{1}=\mathcal{A}$. For $a \in \mathbb{C}$ and $m \in \mathbb{N}, \mathbb{N}_{0}=\mathbb{N} \cup\{0\}, \mathbb{N}=\{1,2, \ldots\}$ let

$$
\mathscr{H}[a, m]=\left\{f \in \mathscr{H}(U): f(z)=a+a_{m} z^{m}+a_{m+1} z^{m+1}+\cdots, z \in U\right\} .
$$

Denote by

$$
K=\left\{f \in \mathcal{A}: \Re \frac{z f^{\prime \prime}(z)}{f^{\prime}(z)}+1>0, z \in U\right\}
$$

the class of normalized convex functions in $U$.

Definition 1 ([5], def. 3.5.1). Let $f$ and $g$ be analytic functions in $U$. We say that the function $f$ is subordinate to the function $g$, if there exists a function $w$, which is analytic in $U$ and $w(0)=0 ;|w(z)|<1 ; z \in U$, such that $f(z)=g(w(z)) ; \forall z \in U$. We denote by $\prec$ the subordination relation. If $g$ is univalent, then $f \prec g$ if and only if $f(0)=g(0)$ and $f(U) \subseteq g(U)$. 
Let $\psi: \mathbb{C}^{3} \times U \rightarrow \mathbb{C}$ be a function and let $h$ be univalent in $U$. If $p$ is analytic in $U$ and satisfies the (second-order) differential subordination

(i) $\psi\left(p(z), z p^{\prime}(z), z^{2} p^{\prime \prime}(z) ; z\right) \prec h(z),(z \in U)$

then $p$ is called a solution of the differential subordination. The univalent function $q$ is called a dominant of the solution of the differential subordination, or more simply a dominant, if $p \prec q$ for all $p$ satisfying $(i)$. A dominant $\widetilde{q}$, which satisfies $\widetilde{q} \prec q$ for all dominants $q$ of $(i)$ is said to be the best dominant of $(i)$. The best dominant is unique up to a rotation of $U$. In order to prove the original results we use the following lemmas.

Lemma 1 (Hallenbeck and Ruscheweyh, [2]). Let $h$ be a convex function with $h(0)=a$, and let $\gamma \in \mathbb{C}^{*}$ be a complex number with $\Re \gamma \geq 0$. If $p \in \mathscr{H}[a, n]$ and

$$
p(z)+\frac{1}{\gamma} z p^{\prime}(z) \prec h(z), \quad z \in U
$$

then

$$
p(z) \prec q(z) \prec h(z), z \in U
$$

where

$$
q(z)=\frac{\gamma}{n z^{\gamma / n}} \int_{0}^{z} h(t) t^{\gamma / n-1} d t, \quad z \in U .
$$

Lemma 2 (Miller and Mocanu, [3]). Let $q$ be a convex function in $U$ and let

$$
h(z)=q(z)+n \alpha z q^{\prime}(z), \quad z \in U
$$

where $\alpha>0$ and $n$ is a positive integer. If

$$
p(z)=q(0)+p_{n} z^{n}+p_{n+1} z^{n+1}+\cdots, \quad z \in U
$$

is holomorphic in $U$ and

$$
p(z)+n \alpha z p^{\prime}(z) \prec h(z), \quad z \in U
$$

then

$$
p(z) \prec q(z)
$$

and this result is sharp.

Definition 2 ([8]). For $f \in \mathscr{A}, n \in \mathbb{N}_{0}$, the Sălăgean differential operator $\mathscr{D}^{n}$ is defined by $\mathscr{D}^{n}: \mathcal{A} \rightarrow \mathcal{A}$,

$$
\begin{gathered}
\mathscr{D}^{0} f(z)=f(z), \\
\cdots \\
\mathscr{D}^{n+1} f(z)=z\left(\mathscr{D}^{n} f(z)\right)^{\prime}, z \in U
\end{gathered}
$$


Remark 1. If $f \in \mathcal{A}$ and $f(z)=z+\sum_{k=2}^{\infty} a_{k} z^{k}$, then

$$
\mathscr{D}^{n} f(z)=z+\sum_{k=2}^{\infty} k^{n} a_{k} z^{k}, z \in U .
$$

Definition 3 ([8]). For $f \in \mathcal{A}, n \in \mathbb{N}_{0}=\mathbb{N} \cup\{0\}, \mathbb{N}=\{1,2, \ldots\}$, the operator $I^{n}$ is defined by

$$
\begin{gathered}
I^{0} f(z)=f(z), \\
\ldots \\
I^{n} f(z)=I\left(I^{n-1} f(z)\right), z \in U
\end{gathered}
$$

Remark 2. If $f \in \mathcal{A}$ and $f(z)=z+\sum_{k=2}^{\infty} a_{k} z^{k}$, then

$$
I^{n} f(z)=z+\sum_{k=2}^{\infty} \frac{a_{k}}{k^{n}} z^{k},
$$

$z \in U,\left(n \in \mathbb{N}_{0}\right)$ and $z\left(I^{n} f(z)\right)^{\prime}=I^{n-1} f(z)$.

Definition 4. Let $\lambda \geq 0, n \in \mathbb{N}$. Denote by $\mathscr{L}^{n}$ the operator given by $\mathscr{L}^{n}: \mathcal{A} \rightarrow \mathcal{A}$,

$$
\mathscr{L}^{n} f(z)=(1-\lambda) \mathscr{D}^{n} f(z)+\lambda I^{n} f(z), z \in U .
$$

Remark 3. If $f \in \mathcal{A}$ and $f(z)=z+\sum_{k=2}^{\infty} a_{k} z^{k}$, then

$$
\mathscr{L}^{n} f(z)=z+\sum_{k=2}^{\infty}\left[k^{n}(1-\lambda)+\lambda \frac{1}{k^{n}}\right] a_{k} z^{k}, z \in U \text {. }
$$

\section{MAIN RESULTS}

Theorem 1. Let $q$ be a convex function, $q(0)=1$ and let $h$ be the function

$$
h(z)=q(z)+z q^{\prime}(z), z \in U .
$$

If $f \in \mathcal{A}, \lambda \geq 0, n \in \mathbb{N}$ and satisfies the differential subordination

$$
\left[\mathscr{L}^{n} f(z)\right]^{\prime} \prec h(z), \quad z \in U
$$

then

and this result is sharp.

$$
\frac{\mathscr{L}^{n} f(z)}{z} \prec q(z), \quad z \in U
$$


Proof. Let

$p(z)=\frac{\mathscr{L}^{n} f(z)}{z}=\frac{z+\sum_{k=2}^{\infty}\left[k^{n}(1-\lambda)+\lambda \frac{1}{k^{n}}\right] a_{k} z^{k}}{z}=1+p_{n} z^{n}+p_{n+1} z^{n+1}+\cdots$

$z \in U$. From (2.2) we have $p \in \mathscr{H}[1,1]$. Let

$$
\mathscr{L}^{n} f(z)=z p(z), z \in U .
$$

Differentiating (2.3), we obtain

$$
\left[\mathscr{L}^{n} f(z)\right]^{\prime}=p(z)+z p^{\prime}(z), z \in U .
$$

Then (2.1) becomes

$$
p(z)+z p^{\prime}(z) \prec h(z), z \in U .
$$

By using Lemma 2, we have

$$
p(z) \prec q(z), z \in U,
$$

i.e.

$$
\frac{\mathscr{L}^{n} f(z)}{z} \prec q(z), \quad z \in U
$$

Remark 4. If $\lambda=0$ we get Theorem 4 from Oros [6] and for $\lambda=1$ we get Theorem 4 from Bălăeți [1].

Example 1. For $\lambda=0, n=1, f \in \mathcal{A}$ we deduce that

$$
f^{\prime}(z)+z f^{\prime \prime}(z) \prec \frac{1}{(1-z)^{2}}, \quad z \in U
$$

implies

$$
f^{\prime}(z) \prec \frac{1}{1-z}, \quad z \in U .
$$

Example 2. For $\lambda=1, n=1, f \in \mathcal{A}$ we deduce that

$$
\frac{f(z)}{z} \prec \frac{1}{(1-z)^{2}}, \quad z \in U
$$

implies

$$
\frac{\int_{0}^{z} f(t) t^{-1} d t}{z} \prec \frac{1}{1-z}, \quad z \in U .
$$


Theorem 2. Let $q$ be a convex function, $q(0)=1$ and let $h$ be the function

$$
h(z)=q(z)+z q^{\prime}(z), z \in U .
$$

If $f \in \mathcal{A}, \lambda \geq 0, n \in \mathbb{N}$ and satisfies the differential subordination

$$
\left(\frac{z \mathscr{L}^{n+1} f(z)}{\mathscr{L}^{n} f(z)}\right)^{\prime} \prec h(z), \quad z \in U
$$

then

and this result is sharp.

$$
\frac{\mathscr{L}^{n+1} f(z)}{\mathscr{L}^{n} f(z)} \prec q(z), \quad z \in U
$$

Proof. Let

$$
p(z)=\frac{\mathscr{L}^{n+1} f(z)}{\mathscr{L}^{n} f(z)}=\frac{z+\sum_{k=2}^{\infty}\left[k^{n+1}(1-\lambda)+\lambda \frac{1}{k^{n+1}}\right] a_{k} z^{k}}{z+\sum_{k=2}^{\infty}\left[k^{n}(1-\lambda)+\lambda \frac{1}{k^{n}}\right] a_{k} z^{k}} .
$$

We have $p^{\prime}(z)=\frac{\left(\mathscr{L}^{n+1} f(z)\right)^{\prime}}{\mathscr{L}^{n} f(z)}-p(z) \frac{\left(\mathscr{L}^{n} f(z)\right)^{\prime}}{\mathscr{L}^{n} f(z)}$ and

$p(z)+z p^{\prime}(z)=\left(\frac{z \mathscr{L}^{n+1} f(z)}{\mathscr{L}^{n} f(z)}\right)^{\prime}$.

Relation (2.6) becomes

$$
p(z)+z p^{\prime}(z) \prec h(z)=q(z)+z q^{\prime}(z), \quad z \in U .
$$

By using Lemma 2 we have

$$
p(z) \prec q(z) \text { i.e. } \frac{\mathscr{L}^{n+1} f(z)}{\mathscr{L}^{n} f(z)} \prec q(z), \quad z \in U .
$$

Theorem 3. Let $q$ be a convex function, $q(0)=1$ and let $h$ be the function

$$
h(z)=q(z)+z q^{\prime}(z), z \in U .
$$

If $f \in \mathcal{A}, \lambda \geq 0, n \in \mathbb{N}$ and satisfies the differential subordination

$$
\left(\mathscr{L}^{n+1} f(z)\right)^{\prime}+\lambda\left[\left(I^{n-1} f(z)\right)^{\prime}-\left(I^{n+1} f(z)\right)^{\prime}\right] \prec h(z), \quad z \in U
$$

then

and this result is sharp.

$$
\left[\mathscr{L}^{n} f(z)\right]^{\prime} \prec q(z), \quad z \in U
$$


Proof. By using the properties of operator $\mathscr{L}^{n}$, we obtain

$$
\mathscr{L}^{n+1} f(z)=(1-\lambda) \mathscr{D}^{n+1} f(z)+\lambda I^{n+1} f(z), z \in U .
$$

Then (2.7) becomes

$$
\left[(1-\lambda) \mathscr{D}^{n+1} f(z)+\lambda I^{n+1} f(z)\right]^{\prime}+\lambda\left[\left(I^{n-1} f(z)\right)^{\prime}-\left(I^{n+1} f(z)\right)^{\prime}\right] \prec h(z), \quad z \in U .
$$

After computation we get

$$
(1-\lambda)\left[\mathscr{D}^{n+1} f(z)\right]^{\prime}+\lambda\left[I^{n-1} f(z)\right]^{\prime} \prec h(z)
$$

or equivalently

$$
(1-\lambda)\left[z\left(\mathscr{D}^{n} f(z)\right)^{\prime}\right]^{\prime}+\lambda\left[z\left(I^{n} f(z)\right)^{\prime}\right]^{\prime} \prec h(z) .
$$

The above relation is equivalent to

$$
(1-\lambda)\left[\left(\mathscr{D}^{n} f(z)\right)^{\prime}+z\left(\mathscr{D}^{n} f(z)\right)^{\prime \prime}\right]+\lambda\left[\left(I^{n} f(z)\right)^{\prime}+z\left(I^{n} f(z)\right)^{\prime \prime}\right] \prec h(z)
$$

or

$$
\left[\mathscr{L}^{n} f(z)\right]^{\prime}+z\left[\mathscr{L}^{n} f(z)\right]^{\prime \prime} \prec h(z), \quad z \in U
$$

Let

$$
\begin{aligned}
p(z)= & (1-\lambda)\left[\mathscr{D}^{n} f(z)\right]^{\prime}+\lambda\left[I^{n} f(z)\right]^{\prime}=\left[\mathscr{L}^{n} f(z)\right]^{\prime}, \quad z \in U \\
& =(1-\lambda)\left[z+\sum_{k=2}^{\infty} k^{n} a_{k} z^{k}\right]^{\prime}+\lambda\left[z+\sum_{k=2}^{\infty} \frac{1}{k^{n}} a_{k} z^{k}\right]^{\prime}= \\
& =(1-\lambda)\left[1+\sum_{k=2}^{\infty} k^{n+1} a_{k} z^{k-1}\right]+\lambda\left[1+\sum_{k=2}^{\infty} \frac{1}{k^{n-1}} a_{k} z^{k-1}\right]= \\
= & 1+\sum_{k=2}^{\infty}\left[k^{n+1}(1-\lambda)+\lambda \frac{1}{k^{n-1}}\right] a_{k} z^{k-1}=1+p_{1} z+p_{2} z^{2}+\cdots
\end{aligned}
$$

In view of (2.11), we deduce that $p \in \mathscr{H}[1,1]$. Using the notation in (2.11), the (2.10) differential subordination becomes

$$
p(z)+z p^{\prime}(z) \prec h(z)=q(z)+z q^{\prime}(z), \quad z \in U .
$$

By using Lemma 2 we have

$$
p(z) \prec q(z) \text { i.e. }\left[\mathscr{L}^{n} f(z)\right]^{\prime} \prec q(z), z \in U .
$$

Remark 5. If $\lambda=0$ we get Theorem 2 from Oros [6] and for $\lambda=1$ we get Theorem 2 from Bălăeţi [1]. 
Example 3. For $\lambda=0, n=1, f \in \mathcal{A}$ we deduce that

$$
f^{\prime}(z)+3 z f^{\prime \prime}(z)+z^{2} f^{\prime \prime \prime}(z) \prec 1+2 z, \quad z \in U
$$

implies

$$
f^{\prime}(z)+z f^{\prime \prime}(z) \prec 1+z, \quad z \in U .
$$

Theorem 4. Let $h \in \mathscr{H}(U)$ such that $h(0)=1$ and

$$
\Re\left[1+\frac{z h^{\prime \prime}(z)}{h^{\prime}(z)}\right]>-\frac{1}{2}, \quad z \in U .
$$

If $f \in \mathcal{A}$ satisfies the differential subordination

$$
\left(\mathscr{L}^{n+1} f(z)\right)^{\prime}+\lambda\left[\left(I^{n-1} f(z)\right)^{\prime}-\left(I^{n+1} f(z)\right)^{\prime}\right] \prec h(z), \quad z \in U
$$

then

$$
\left[\mathscr{L}^{n} f(z)\right]^{\prime} \prec q(z), \quad z \in U
$$

where $q$ is given by $q(z)=\frac{1}{z} \int_{0}^{z} h(t) d t$. The function $q$ is convex and is the best dominant.

Proof. If we use the differential subordination technique we can see that the function $g$ is convex.[3], p. 66 By using (2.11) we obtain

$$
\left(\mathscr{L}^{n+1} f(z)\right)^{\prime}+\lambda\left[\left(I^{n-1} f(z)\right)^{\prime}-\left(I^{n+1} f(z)\right)^{\prime}\right]=p(z)+z p^{\prime}(z), \quad z \in U
$$

Then (2.12) becomes

$$
p(z)+z p^{\prime}(z) \prec h(z), \quad z \in U .
$$

Since $p \in \mathscr{H}[1,1]$, we deduce that $p(z) \prec q(z)$, i.e.

$$
\left[\mathscr{L}^{n} f(z)\right]^{\prime} \prec q(z)=\frac{1}{z} \int_{0}^{z} h(t) d t, \quad z \in U
$$

and $q$ is the best dominant.

Remark 6. If $\lambda=0$ we get Theorem 3 from Oros [6].

Example 4. For $\lambda=0, n=0, h(z)=\frac{1+z}{1-z}$ we deduce that

$$
f^{\prime}(z)+z f^{\prime \prime}(z) \prec \frac{1+z}{1-z}, \quad z \in U,
$$

implies

$$
f^{\prime}(z) \prec 1-\frac{2}{z} \ln (1-z), \quad z \in U \text {. }
$$


Theorem 5. Let $h \in \mathscr{H}(U)$ such that $h(0)=1$ and

$$
\Re\left[1+\frac{z h^{\prime \prime}(z)}{h^{\prime}(z)}\right]>-\frac{1}{2}, \quad z \in U .
$$

If $f \in \mathcal{A}$ satisfies the differential subordination

$$
\left[\mathscr{L}^{n} f(z)\right]^{\prime} \prec h(z), \quad z \in U
$$

then

$$
\frac{\mathscr{L}^{n} f(z)}{z} \prec q(z), \quad z \in U
$$

where $q$ is given by $q(z)=\frac{1}{z} \int_{0}^{z} h(t) d t$. The function $q$ is convex and is the best dominant.

Proof. If we use the differential subordination technique we can see that the function $g$ is convex. [3], p. 66. Differentiating both sides in (2.2) we obtain

$$
\left[\mathscr{L}^{n} f(z)\right]^{\prime}=p(z)+z p^{\prime}(z), \quad z \in U
$$

Then (2.13) becomes

$$
p(z)+z p^{\prime}(z) \prec h(z), \quad z \in U .
$$

Since $p \in \mathscr{H}[1,1]$, we deduce that $p(z) \prec q(z)$, i.e.

$$
\frac{\mathscr{L}^{n} f(z)}{z} \prec q(z)=\frac{1}{z} \int_{0}^{z} h(t) d t, \quad z \in U
$$

and $q$ is the best dominant.

Remark 7. If $\lambda=0$ we get Theorem 5 from Oros [6] and for $\lambda=1$ we get Theorem 5 from Bălăeţi [1].

Example 5. For $\lambda=0, n=1, h(z)=\frac{1}{(1+z)^{2}}$ we deduce that

$$
f^{\prime}(z) \prec \frac{1}{(1+z)^{2}}, \quad z \in U,
$$

implies

We get the same result as [4].

$$
\frac{f(z)}{z} \prec \frac{1}{1+z}, \quad z \in U .
$$

Definition 5 ([7], [9], [1], [6]). If $0 \leq \beta<1$ and $n \in \mathbb{N}$, we let $L_{n}^{m}(\beta)$ stand for the class of functions $f \in \mathcal{A}_{m}$, which satisfy the inequality

$$
\Re\left[\mathscr{L}^{n} f(z)\right]^{\prime}>\beta, \quad(z \in U) .
$$

Remark 8. For $n=0$ we obtain $\Re f^{\prime}(z)>\beta$. 
Theorem 6. The set $L_{n}^{m}(\beta)$ is convex.

Proof. Let the function

$$
f_{i}(z)=z+\sum_{k=2}^{\infty} a_{k_{i}} z^{k}, \quad i=1,2 \quad z \in U
$$

be in the class $L_{n}^{m}(\beta)$. It is sufficient to show that the function

$$
h(z)=\mu_{1} f_{1}(z)+\mu_{2} f_{2}(z)
$$

with $\mu_{1}, \mu_{2} \geq 0$ and $\mu_{1}+\mu_{2}=1$ is in $L_{n}(\beta)$. Since

$$
h(z)=z+\sum_{k=2}^{\infty}\left(\mu_{1} a_{k_{1}}+\mu_{2} a_{k_{2}}\right) z^{k}, z \in U
$$

then

$$
\mathscr{L}^{n} h(z)=z+\sum_{k=2}^{\infty}\left[k^{n}(1-\lambda)+\lambda \frac{1}{k^{n}}\right]\left(\mu_{1} a_{k_{1}}+\mu_{2} a_{k_{2}}\right) z^{k}, \quad z \in U .
$$

Differentiating (2.14), we get

$$
\left[\mathscr{L}^{n} h(z)\right]^{\prime}=1+\sum_{k=2}^{\infty}\left[k^{n+1}(1-\lambda)+\lambda \frac{1}{k^{n-1}}\right]\left(\mu_{1} a_{k_{1}}+\mu_{2} a_{k_{2}}\right) z^{k-1}
$$

Hence

$$
\begin{gathered}
\Re\left[\mathscr{L}^{n} h(z)\right]^{\prime}=1+\Re\left\{\mu_{1} \sum_{k=2}^{\infty}\left[k^{n+1}(1-\lambda)+\lambda \frac{1}{k^{n-1}}\right] a_{k_{1}} z^{k-1}\right\}+ \\
+\Re\left\{\mu_{2} \sum_{k=2}^{\infty}\left[k^{n+1}(1-\lambda)+\lambda \frac{1}{k^{n-1}}\right] a_{k_{2}} z^{k-1}\right\} .
\end{gathered}
$$

Since $f_{1}, f_{2} \in L_{n}^{m}(\beta)$, we obtain

$$
\mathfrak{R}\left\{\mu_{i} \sum_{k=2}^{\infty}\left[k^{n+1}(1-\lambda)+\lambda \frac{1}{k^{n-1}}\right] a_{k_{i}} z^{k-1}\right\}>\mu_{i}(\beta-1), \quad i=1,2 .
$$

Using (2.16) we get from (2.15)

$$
\mathfrak{R}\left[\mathscr{L}^{n} h(z)\right]^{\prime}>1+\mu_{1}(\beta-1)+\mu_{2}(\beta-1),
$$

and since $\mu_{1}+\mu_{2}=1$, we deduce

$$
\Re\left[\mathscr{L}^{n} h(z)\right]^{\prime}>\beta, \quad(z \in U)
$$

i.e. $L_{n}^{m}(\beta)$ is convex. 
Theorem 7. If $0 \leq \beta<1$ and $m, n \in \mathbb{N}$ then we have

$$
L_{n}^{m}(\beta) \subset L_{n+1}^{m}(\delta),
$$

where $\delta(\beta, m)=2 \beta-1+2(1-\beta) \frac{1}{m} \sigma\left(\frac{1}{m}\right)$ and $\sigma(x)=\int_{0}^{z} \frac{t^{x-1}}{1+t} d t$. The result is sharp.

Proof. Assume that $f \in L_{n}^{m}(\beta)$. Let $\mathscr{L}^{n} f(z)=z p(z), z \in U$. Differentiating, we obtain

$$
\left[\mathscr{L}^{n} f(z)\right]^{\prime}=p(z)+z p^{\prime}(z), z \in U .
$$

Since $f \in L_{n}^{m}(\beta)$, from Definition 5 we have

$$
\Re\left(p(z)+z p^{\prime}(z)\right)>\beta, z \in U
$$

which is equivalent to

$$
p(z)+z p^{\prime}(z) \prec \frac{1+(2 \beta-1) z}{1+z} \equiv h(z), z \in U
$$

By using Lemma 1, we have:

$$
p(z) \prec q(z) \prec h(z), z \in U,
$$

where

$$
\begin{gathered}
q(z)=\frac{1}{m z^{\frac{1}{m}}} \int_{0}^{z} \frac{1+(2 \beta-1) t}{1+t} t^{\frac{1}{m}-1} d t= \\
=\frac{1}{m z^{\frac{1}{m}}} \int_{0}^{z}\left[2 \beta-1+2(1-\beta) \frac{1}{1+t}\right] t^{\frac{1}{m}-1} d t= \\
=\frac{1}{m z^{\frac{1}{m}}} \int_{0}^{z}(2 \beta-1) t^{\frac{1}{m}-1} d t+\frac{2(1-\beta)}{m z^{\frac{1}{m}}} \int_{0}^{z} \frac{t^{\frac{1}{m}-1}}{1+t} d t= \\
=2 \beta-1+2(1-\beta) \frac{1}{m} \sigma\left(\frac{1}{m}\right) \frac{1}{z^{\frac{1}{m}}}, z \in U .
\end{gathered}
$$

The function $q$ is convex and is the best dominant. From $p(z) \prec q(z)$ follows that

$$
\Re p(z)>\Re q(1)=\delta(\beta, m)=2 \beta-1+2(1-\beta) \frac{1}{m} \sigma\left(\frac{1}{m}\right),
$$

from which we deduce that $L_{n}^{m}(\beta) \subset L_{n+1}^{m}(\delta)$.

Remark 9. If $\lambda=0$ we get Theorem 1 from Oros [6] and for $\lambda=1$ we get Theorem 1 from Bălăeţi [1]. 
Theorem 8. Let $q$ be a convex function in $U$ with $q(0)=1$ and let

$$
h(z)=q(z)+\frac{1}{c+2} z q^{\prime}(z), z \in U,
$$

where $c$ is a complex number, with $\Re c>-2$.

If $f \in L_{n}^{m}(\beta)$ and $F=I_{c}(f)$, where

$$
F(z)=I_{c}(f)(z)=\frac{c+2}{z^{c+1}} \int_{0}^{z} t^{c} f(t) d t, \Re c>-2,
$$

then

implies

$$
\left[\mathscr{L}^{n} f(z)\right]^{\prime} \prec h(z), \quad z \in U
$$

and this result is sharp.

$$
\left[\mathscr{L}^{n} F(z)\right]^{\prime} \prec q(z), \quad z \in U
$$

Proof. From (2.17), we have

$$
z^{c+1} F(z)=(c+2) \int_{0}^{z} t^{c} f(t) d t, \Re c>-2, \quad z \in U .
$$

Differentiating, with respect to $\mathrm{z}$, we obtain

$$
(c+1) F(z)+z F^{\prime}(z)=(c+2) f(z), \quad z \in U
$$

and

$$
(c+1) \mathscr{L}^{n} F(z)+z\left[\mathscr{L}^{n} F(z)\right]^{\prime}=(c+2) \mathscr{L}^{n} f(z), \quad z \in U .
$$

Differentiating (2.20), we obtain

$$
\left[\mathscr{L}^{n} F(z)\right]^{\prime}+\frac{z}{c+2}\left[\mathscr{L}^{n} F(z)\right]^{\prime \prime}=\left[\mathscr{L}^{n} f(z)\right]^{\prime}, \quad z \in U .
$$

Using (2.21), the differential subordination (2.18) becomes

$$
\left[\mathscr{L}^{n} F(z)\right]^{\prime}+\frac{1}{c+2} z\left[\mathscr{L}^{n} F(z)\right]^{\prime \prime} \prec h(z)=q(z)+\frac{1}{c+2} z q^{\prime}(z), \quad z \in U .
$$

Let

$$
\begin{gathered}
p(z)=\left[\mathscr{L}^{n} F(z)\right]^{\prime}=\left\{z+\sum_{k=2}^{\infty}\left[k^{n}(1-\lambda)+\lambda \frac{1}{k^{n}}\right] a_{k} z^{k}\right\}^{\prime}= \\
=1+p_{1} z+p_{2} z^{2}+\cdots, \quad z \in U, \quad p \in \mathscr{H}[1,1] .
\end{gathered}
$$

Replacing (2.23) in (2.22) we obtain

$$
p(z)+\frac{1}{c+2} z p^{\prime}(z) \prec h(z)=q(z)+\frac{1}{c+2} z q^{\prime}(z), \quad z \in U,
$$

Using Lemma 1 , we obtain $p(z) \prec q(z)$ i.e.

$$
\left[\mathscr{L}^{n} F(z)\right]^{\prime} \prec q(z), \quad z \in U,
$$

and $\mathrm{q}$ is the best dominant. 
Remark 10. If $\lambda=0$ we get Theorem 2.2 from Tăut et alii [9].

Example 6. If we take $c=1+2 i$ and $q(z)=\frac{1+z}{1-z}$ then $h(z)=\frac{\left(1-z^{2}\right)(3+2 i)+2 z}{(3+2 i)(1-z)^{2}}$.

From Theorem 8 we deduce

implies

$$
\left[\mathscr{L}^{n} f(z)\right]^{\prime} \prec \frac{\left(1-z^{2}\right)(3+2 i)+2 z}{(3+2 i)(1-z)^{2}}, z \in U
$$

where $F$ is given by (2.17).

$$
\left[\mathscr{L}^{n} F(z)\right]^{\prime} \prec \frac{1+z}{1-z}, \quad z \in U
$$

\section{REFERENCES}

[1] C. M. Bălăeți, “A general class of holomorphic functions defined by integral operator," General Mathematics., vol. 18, no. 2, pp. 59-69, 2010.

[2] D. Hallenbeck and S. Ruscheweyh, "Subordination by convex functions," Proc. Amer. Math. Soc., vol. 52, pp. 191-195, 1975.

[3] S. S. Miller and P. T. Mocanu, Differential Subordinations. Theory and Applications. Marcel Dekker Inc., New York, Basel, 2000.

[4] S. S. Miller, P. T. Mocanu , and M. O. Reade, "Subordination-preserving integral operators," Trans. Amer. Math. Soc., vol. 283, pp. 605-615, 1984.

[5] P. T. Mocanu, T. Bulboacă, and G. S. Sălăgean, The geometric theory of univalent functions. ClujNapoca: Casa Cărţii de Ştiinţă, 1999.

[6] G. Oros , "On a class of holomorphic functions defined by Sălăgean differential operator," Complex Variables., vol. 50, no. 4, pp. 257-264, 2005.

[7] G. Oros and G. I. Oros , "A Class of Holomorphic Function II," Libertas Math., vol. 23, pp. 65-68, 2003.

[8] G. S. Sălăgean, "Subclasses of univalent functions," Lecture Notes in Math. (Springer Verlag), vol. 1013, pp. 362-372, 1983.

[9] A. O. Tăut, G. I. Oros, and R. Şendruţiu, "On a class of univalent functions defined by Sǎlăgean differential operator," Banach J. Math. Anal., vol. 3, no. 1, pp. 61-67, 2009.

Author's address

\section{Á. O. Páll-Szabó}

Babeş-Bolyai University, Cluj-Napoca, Romania

E-mail address: pallszaboagnes@math. ubbcluj.ro 